\title{
Internal Leakage Fault Detection and Tolerant Control of Single-Rod Hydraulic Actuators
}

\author{
Jianyong Yao, Guichao Yang, and Dawei Ma \\ School of Mechanical Engineering, Nanjing University of Science and Technology, Nanjing 210094, China \\ Correspondence should be addressed to Jianyong Yao; jerryyao.buaa@gmail.com
}

Received 24 December 2013; Accepted 31 January 2014; Published 13 March 2014

Academic Editor: Weichao Sun

Copyright (c) 2014 Jianyong Yao et al. This is an open access article distributed under the Creative Commons Attribution License, which permits unrestricted use, distribution, and reproduction in any medium, provided the original work is properly cited.

\begin{abstract}
The integration of internal leakage fault detection and tolerant control for single-rod hydraulic actuators is present in this paper. Fault detection is a potential technique to provide efficient condition monitoring and/or preventive maintenance, and fault tolerant control is a critical method to improve the safety and reliability of hydraulic servo systems. Based on quadratic Lyapunov functions, a performance-oriented fault detection method is proposed, which has a simple structure and is prone to implement in practice. The main feature is that, when a prescribed performance index is satisfied (even a slight fault has occurred), there is no fault alarmed; otherwise (i.e., a severe fault has occurred), the fault is detected and then a fault tolerant controller is activated. The proposed tolerant controller, which is based on the parameter adaptive methodology, is also prone to realize, and the learning mechanism is simple since only the internal leakage is considered in parameter adaptation and thus the persistent exciting (PE) condition is easily satisfied. After the activation of the fault tolerant controller, the control performance is gradually recovered. Simulation results on a hydraulic servo system with both abrupt and incipient internal leakage fault demonstrate the effectiveness of the proposed fault detection and tolerant control method.
\end{abstract}

\section{Introduction}

Hydraulic systems have been used in industry in a wide number of applications, including robotics and manipulators [1], machine tools [2], active suspension systems [3, 4], hydraulic positioning systems [5-8], and hydraulic load simulators [9-11], by virtue of their small size-to-power ratios, high response, high stiffness, and high load capability. Due to their ever increasing use in industry and elsewhere, it is essential that such systems should be made more reliable and safe in operation. The dynamic characteristics of hydraulic systems, however, suffer various faults [12] due to wear, battle damage, and/or unexpected failures of the system components. Therefore, fault detection and diagnosis (FDD) and fault tolerant control (FTC) of hydraulic systems have received more and more attentions.

FDD is a potential technique to provide efficient condition monitoring and/or preventive maintenance for hydraulic systems but is also a very challenging task, as it is extremely difficult to model such systems precisely owing to their nonlinear dynamics [13] and uncertainties [14]. Condition monitoring of hydraulic systems is therefore very useful in the early detection of component failure which would lead to better operational safety and economy. This has led to the increasing trend towards integrating elements of FDD as part of a control system design [15]. In order to develop FDD algorithms for hydraulic systems, a number of different approaches have been proposed in the literature. These include methods based on hardware redundancy [16], parameter estimation methods which utilize linearized models [17] and Volterra models [18], robust observer based methods using nonlinear system models $[19,20]$, and an informative statistical study [21]. In order to account for the presence of parametric uncertainties, Extended Kalman Filters [22] and adaptive observers [23] which estimate both the states and parameters of the system have found application in fault detection for hydraulic systems. To consider both the parametric uncertainties and the uncertain nonlinearities in hydraulic servo systems, Garimella and Yao [24] proposed a model based detection technique based on a nonlinear adaptive robust observer (ARO) [25]. State and parameter 
estimation are both utilized for fault detection in those papers with online PE condition monitoring.

On the other hand, fault tolerant control for electrohydraulic systems has received relatively less attention so far. But, with the increase in demand for robustness and reliability, it is fast becoming an active area of research. In [18], the authors present a fault accommodation (FA) technique based on the linear model of the system. Using the FDD results presented in [24], Gayaka and Yao [26] present a comprehensive scheme for FA which is based on adaptive robust philosophy [27], taking into account various uncertainties present in the hydraulic systems.

The internal leakage is a typical fault in hydraulic systems, which is difficult to measure directly, and will decrease the loop gain and increase the effective damping [28], thus degrading the performance of hydraulic systems. Fault detection methods for internal leakage based on Extended Kalman Filter [29] and wavelet analysis techniques [30] have been developed for double-rod hydraulic actuators. Robust passive fault tolerant control based on quantitative feedback theory (QFT) techniques $[31,32]$ has been widely investigated, but as pointed in [33], passive FTC is often used to save time until a more sophisticated active algorithm enters into the system. In addition, some performance has to be given up by the passive FTC scheme.

For single-rod hydraulic actuators, integrating fault detection and active fault tolerant control for internal leakage fault, which will be focused on in this paper, remains sparse. In contrast to the double-rod hydraulic actuator, the areas of the two chambers of a single-rod hydraulic actuator are different. As a result, the two dynamic equations relating the pressure changes in the two chambers to the servovalve opening cannot be combined into a single equation that relates the load pressure to the valve opening. This complicates the fault detection and tolerant controller design. In this paper, a normal nonlinear robust backstepping controller is firstly developed based on the nominal nonlinear system model, and a prescribed performance index is derived. In the fault detection module, the derived performance index is used as the fault threshold to detect the fault. That is to say, under normal condition, that is, no fault occur, the system performance is dominated by the derived performance index; if a slight leakage has occurred, the designed normal robust controller can cover this fault and the prescribed performance index is also respected. In this case, the control performance is still guaranteed though a leakage fault has occurred; thus the fault is not necessary to be alarmed and detected, the system continues to function under the normal robust controller; if a severe leakage has happened and the prescribed performance index is thus violated, then the fault is detected. Due to only internal leakage fault being considered in this paper, the fault diagnosis module is omitted, and then a fault tolerant controller is activated. The active fault tolerant controller is developed based on the adaptive control philosophy, as the internal leakage can be modeled as a parametric variation. The fault tolerant controller only needs to change a parameter in the normal robust controller and thus has a simple structure and can be easily implemented. The learning process is operated based

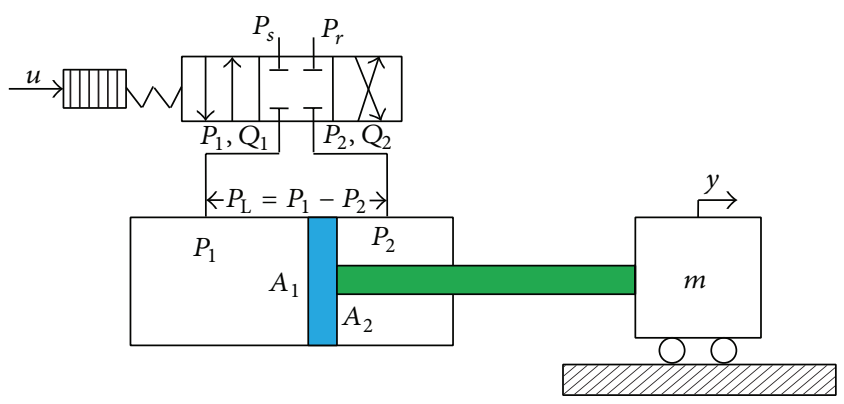

FIGURE 1: Schematic diagram of a single-rod hydraulic actuator system.

on the PE condition which is monitored online to guarantee the excellent convergence of parameter adaptation. After the activation of the FTC controller, the system performance is gradually recovered and the leakage level can be provided for system maintenance.

Extensive simulation results have been obtained for the motion control of a hydraulic system. These results verify the effectiveness of the proposed fault detection and the fault tolerant controller.

The remainder of this paper is organized as follows. Problem formulation and system model are present in Section 2. Normal robust controller, fault detection module, and fault tolerant controller are designed in Section 3. The effectiveness of the proposed fault detection and tolerant control scheme is demonstrated in Section 4, by simulation results. Section 5 gives some conclusions.

\section{Problem Formulation and Dynamic Models}

2.1. Nominal System Models. The system under consideration is depicted in Figure 1. The goal is to have the inertia load to track any specified motion trajectory as closely as possible in spite of various model uncertainties and/or internal leakage fault.

The dynamics of the inertia load can be described by

$$
\begin{gathered}
m \ddot{y}=P_{1} A_{1}-P_{2} A_{2}-F(y, \dot{y}, t), \\
F(y, \dot{y}, t)=F_{f}+F_{e},
\end{gathered}
$$

where $m$ and $y$ represent the mass and the displacement of the load, respectively, $P_{1}$ and $P_{2}$ are the pressures inside the two chambers of the cylinder, $A_{1}$ and $A_{2}$ are the ram areas of the two chambers, and $F$ is the lumped effect of uncertain nonlinearities such as friction $F_{f}$ and external disturbance $F_{e}$ (such as cutting force in machining, hinge moment in aircraft actuation systems). While there have been many friction models proposed [34], a simple and often adequate approach is to regard the friction force as a static nonlinear function of the velocity, which is given by

$$
F_{f}(\dot{y})=B \dot{y}+F_{s}(\dot{y})
$$


where $B$ represents the combined coefficient of the modeled damping and viscous friction forces and $F_{s}$ is the nonlinear term that can be modeled as [34]

$$
F_{s}(\dot{y})=\left[f_{c}+\left(f_{s}-f_{c}\right) e^{-\left|\dot{y} / \dot{y}_{v}\right|^{\sigma}}\right] \operatorname{sgn}(\dot{y})
$$

where $f_{c}, f_{s}$ represent the level of Coulomb friction and stiction friction, respectively, $\dot{y}_{v}, \sigma$ are empirical parameters used to describe the Stribeck effect, and $\operatorname{sgn}(\cdot)$ is the sign function.

The pressure dynamics in the two chambers can be written as [13]

$$
\begin{aligned}
& \dot{P}_{1}=\frac{\beta_{1}}{V_{1}}\left(-A_{1} \dot{y}-q_{\text {leak }}+Q_{1}\right), \\
& \dot{P}_{2}=\frac{\beta_{2}}{V_{2}}\left(A_{2} \dot{y}+q_{\text {leak }}-Q_{2}\right),
\end{aligned}
$$

where $V_{1}=V_{01}+A_{1} y$ and $V_{2}=V_{02}-A_{2} y$ are the total control volume of the first and second chamber, respectively, $V_{01}$ and $V_{02}$ are the original total control volume of the two cylinder chambers, respectively, $\beta_{1}$ and $\beta_{2}$ are the effective bulk modulus of the two cylinder chambers, respectively, and $q_{\text {leak }}$ is the total internal leakage of the actuator due to pressure and can be modeled as follows in the normal case [13]:

$$
q_{\text {leak }}=C_{l} P_{L}
$$

where $C_{l}$ is the normal coefficient of the internal leakage, $P_{L}=$ $P_{1}-P_{2}$ is the load pressure of the hydraulic cylinder, $Q_{1}$ is the supplied flow rate to the forward chamber, and $Q_{2}$ is the return flow rate of the return chamber. $Q_{1}$ and $Q_{2}$ are related to the spool valve displacement of the servovalve, $x_{v}$, by [13]

$$
\begin{aligned}
& Q_{1}=k_{q 1} x_{v}\left[s\left(x_{v}\right) \sqrt{P_{s}-P_{1}}+s\left(-x_{v}\right) \sqrt{P_{1}-P_{r}}\right], \\
& Q_{2}=k_{q 2} x_{v}\left[s\left(x_{v}\right) \sqrt{P_{2}-P_{r}}+s\left(-x_{v}\right) \sqrt{P_{s}-P_{2}}\right],
\end{aligned}
$$

where

$$
k_{q 1}=C_{d} w_{1} \sqrt{\frac{2}{\rho}}, \quad k_{q 2}=C_{d} w_{2} \sqrt{\frac{2}{\rho}}
$$

$s(*)$ is defined as

$$
s(*)= \begin{cases}1, & \text { if } * \geq 0 \\ 0, & \text { if } *<0\end{cases}
$$

where $C_{d}$ is the discharge coefficient, $w_{1}$ and $w_{2}$ are the spool valve area gradients, $\rho$ is the density of oil, $P_{s}$ is the supply pressure of the fluid, and $P_{r}$ is the return pressure. As discussed in some works in the literature, the spool valve displacement $x_{v}$ can be related to the control input $u$ by a firstorder system [35] given by

$$
\tau_{v} \dot{x}_{v}=-x_{v}+k_{i} u
$$

where $\tau_{v}$ and $k_{i}$ are the time constant and gain of the servovalve, respectively, or a second-order system [31] given by

$$
\ddot{x}_{v}=-\omega_{v}^{2} x_{v}-2 \zeta_{v} \omega_{v} \dot{x}_{v}+k_{i} \omega_{v}^{2} u
$$

where $\omega_{v}$ and $\zeta_{v}$ represent the servovalve natural frequency and damping ratio, respectively.

Thus, the nominal system models can be represented by the inertia load dynamics (1) (3), pressure dynamics $(4) \sim(8)$, and the servovalve dynamic (9) or (10).

2.2. Internal Leakage Fault Models. If an internal leakage fault has occurred (i.e., the actuator piston seal has been defective), as suggested by Thompson et al. [36] and widely used in [28, $31,32]$, the rate of fluid flow across a faulty actuator piston seal can be modeled as a turbulent orifice flow. Combined with the normal internal leakage, the $q_{\text {leak }}$ in (5) can be expressed by

$$
q_{\text {leak }}=C_{l} P_{L}+\eta\left(t-T_{f}\right) C_{t} \sqrt{\left|P_{L}\right|} \operatorname{sgn}\left(P_{L}\right),
$$

where $C_{t}$ is the leakage coefficient used to set the severity of the internal leakage fault in the following simulation and $\eta\left(t-T_{f}\right)$ represents the time profile with $T_{f}$ being the time of occurrence of the fault and is written in the following form [37]:

$$
\eta\left(t-T_{f}\right)= \begin{cases}0 & \text { if } t<T_{f} \\ 1-e^{-\mu\left(t-T_{f}\right)} & \text { if } t \geq T_{f}\end{cases}
$$

where $\mu>0$ denotes the rate of fault evolution. Small value of $\mu$ characterizes slowly developing fault, also known as incipient fault. For large value of $\mu$, the time profile $\eta$ approaches a step function, which models the abrupt fault.

Given the desired motion trajectory $y_{d}(t)=x_{1 d}(t)$, our objective is to synthesize a control input $u$ such that the output $y$ tracks $y_{d}(t)$ as closely as possible in spite of model uncertainties and/or internal leakage fault. Meanwhile, a fault detection module is designed to detect the severe internal leakage fault and a fault tolerant controller is synthesized to recover the control performance gradually in the presence of the severe fault.

For the desired motion trajectory, we have the following practical assumption.

Assumption 1. The desired position $y_{d}(t)$ is $C^{3}$ continuous and bounded.

\section{Fault Detection and Controller Design}

3.1. Synthesizing Design Model and Issues to Be Addressed. For the controller design, a suitable design model can simplify the controller's implementation; thus the dynamics of the inertia load can be rewritten as

$$
m \ddot{y}=P_{1} A_{1}-P_{2} A_{2}-B \dot{y}-A_{f} S_{f}(\dot{y})-\tilde{d},
$$

where $\tilde{d} \triangleq F_{e}+F_{s}-A_{f} S_{f}(\dot{y})$ is the lumped unmodeled uncertain nonlinearity and $A_{f} S_{f}$ is used to approximate the 
nonlinear Coulomb friction, in which $A_{f}$ is the amplitude of the Coulomb friction and the $S_{f}$ is a continuous shape function to capture the switching characteristics of the Coulomb friction with respect to the system velocity.

Although the effects of servovalve dynamics have been included in some works in the literature, this requires an additional sensor to obtain the spool position and only minimal performance improvement can be achieved for positioning tracking, so many researchers neglect servovalve dynamics [6]. Since a high-response servovalve is used here, it is assumed that the control applied to the servovalve is directly proportional to the spool position; then the following equation is given: $x_{v}=k_{i} u$. Thus, from (8), $s\left(x_{v}\right)=s(u)$.

Therefore (6) can be transformed to

$$
\begin{aligned}
& Q_{1}=g_{1} u R_{1}, \\
& Q_{2}=g_{2} u R_{2},
\end{aligned}
$$

where $g_{1}=k_{q 1} k_{i}, g_{2}=k_{q 2} k_{i}$, and

$$
\begin{aligned}
& R_{1}=s(u) \sqrt{P_{s}-P_{1}}+s(-u) \sqrt{P_{1}-P_{r}} \\
& R_{2}=s(u) \sqrt{P_{2}-P_{r}}+s(-u) \sqrt{P_{s}-P_{2}} .
\end{aligned}
$$

Assumption 2. For simplification, the servovalve used here is symmetric and then $g=g_{1}=g_{2}=k_{q 1} k_{i}=k_{q 2} k_{i}$. Since high pressure supply is used here and so the effective bulk modulus in the two chambers are large and change very small, assume $\beta_{e}=\beta_{1}=\beta_{2}$. In a practical hydraulic system under normal working condition, $P_{1}$ and $P_{2}$ are both bounded by $0<P_{r}<P_{1}<P_{s}, 0<P_{r}<P_{2}<P_{s}[6]$.

Define the state variables as $x=\left[x_{1}, x_{2}, x_{3}, x_{4}\right]^{T} \triangleq$ $\left[y, \dot{y}, P_{1}, P_{2}\right]^{T}$; the entire design model including (13), (4), (11), (12), (14), and (15) can be expressed in a state-space form as follows:

$$
\begin{aligned}
& \dot{x}_{1}=x_{2}, \\
& \dot{x}_{2}=\frac{1}{m}\left[A_{1} x_{3}-A_{2} x_{4}-B x_{2}-A_{f} S_{f}\left(x_{2}\right)-\tilde{d}\right] \\
& \dot{x}_{3}=\frac{\beta_{e}}{V_{1}}\left[-A_{1} x_{2}-C_{l}\left(x_{3}-x_{4}\right)-\eta\left(t-T_{f}\right) C_{t} \sqrt{\left|x_{3}-x_{4}\right|}\right. \\
& \left.\quad \times \operatorname{sgn}\left(x_{3}-x_{4}\right)+g R_{1} u\right], \\
& \dot{x}_{4}=\frac{\beta_{e}}{V_{2}}\left[A_{2} x_{2}+C_{l}\left(x_{3}-x_{4}\right)+\eta\left(t-T_{f}\right) C_{t} \sqrt{\left|x_{3}-x_{4}\right|}\right. \\
& \left.\quad \times \operatorname{sgn}\left(x_{3}-x_{4}\right)-g R_{2} u\right] .
\end{aligned}
$$

In order to make system (16) fall into the classic class of system known as strict feedback form to use the backstepping method [38] appropriately, define a new state variable as $\bar{x}_{3}=A_{1} x_{3}-A_{2} x_{4}[5,6,14]$; then, the state-space equation (16) is transformed as

$$
\begin{aligned}
& \dot{x}_{1}=x_{2}, \\
& \dot{x}_{2}=\frac{1}{m}\left[\bar{x}_{3}-B x_{2}-A_{f} S_{f}\left(x_{2}\right)-\tilde{d}\right], \\
& \dot{\bar{x}}_{3}=f_{1}(x) u-f_{2}(x)-\eta\left(t-T_{f}\right) C_{t} f_{3}(x),
\end{aligned}
$$

where

$$
\begin{aligned}
f_{1}(x)= & \left(\frac{A_{1} R_{1}}{V_{1}}+\frac{A_{2} R_{2}}{V_{2}}\right) \beta_{e} g \\
f_{2}(x)= & \frac{A_{1}}{V_{1}} \beta_{e}\left[A_{1} x_{2}+C_{l}\left(x_{3}-x_{4}\right)\right] \\
& +\frac{A_{2}}{V_{2}} \beta_{e}\left[A_{2} x_{2}+C_{l}\left(x_{3}-x_{4}\right)\right], \\
f_{3}(x)= & \left(\frac{A_{1}}{V_{1}}+\frac{A_{2}}{V_{2}}\right) \beta_{e} \sqrt{\left|x_{3}-x_{4}\right|} \operatorname{sgn}\left(x_{3}-x_{4}\right) .
\end{aligned}
$$

In this paper, the full state feedback is employed and $P_{s}$, $P_{r}$ are measured for nonlinear control. In general, the system is subjected to parametric uncertainties due to the variations of $m, B, \beta_{e}, C_{l}, C_{t}$, and so on. Many adaptive controllers for hydraulic systems have been proposed to tackle these parameter uncertainties; however, we only consider the parametric uncertainty due to the internal leakage fault, that is, the parameter $C_{t}$ for better fault detection and tolerant control. In many applications, for example, aircraft actuation systems, machine tools, testing instruments, and so on, this is not a strong assumption as the other parameters can be identified off-line, and the identification errors can be lumped into uncertain nonlinearities. Define the unknown parameter $\theta=C_{t}$; thus the state-space equation (17) is transformed to

$$
\begin{aligned}
& \dot{x}_{1}=x_{2}, \\
& \dot{x}_{2}=\frac{1}{m}\left[\bar{x}_{3}-B x_{2}-A_{f} S_{f}\left(x_{2}\right)-\tilde{d}\right], \\
& \dot{\bar{x}}_{3}=f_{1}(x) u-f_{2}(x)-\eta\left(t-T_{f}\right) \theta f_{3}(x) .
\end{aligned}
$$

For most applications, the extent of the internal leakage fault and uncertain nonlinearities are known. Thus the following practical assumption is made $[5,14]$.

Assumption 3. Parametric uncertainty and uncertain nonlinearities satisfy

$$
\begin{gathered}
\theta \in \Omega_{\theta} \triangleq\left\{\theta: \theta_{\min } \leq \theta \leq \theta_{\max }\right\}, \\
|\tilde{d}| \leq \delta_{d}(t),
\end{gathered}
$$

where $\theta_{\min }, \theta_{\max }$, and $\delta_{d}(t)$ are known.

Since $x_{1}$ is the displacement of the cylinder, noting (15) and Assumption 2, the following inequation always holds in hydraulic control systems

$$
f_{1}(x)>0, \quad \forall x .
$$


3.2. Discontinuous Projection Mapping. Let $\hat{\theta}$ denote the estimate of unknown parameter $\theta$ and $\widetilde{\theta}$ the estimation error (i.e., $\widetilde{\theta}=\widehat{\theta}-\theta$ ). Viewing (20a), a discontinuous projection can be defined as [14]

$$
\operatorname{Proj}_{\widehat{\theta}}(\bullet)= \begin{cases}0 & \text { if } \widehat{\theta}=\theta_{\text {max }} \text { and } \bullet>0, \\ 0 & \text { if } \widehat{\theta}=\theta_{\text {min }} \text { and } \bullet<0 \\ \bullet & \text { otherwise }\end{cases}
$$

for an adaptation law given by $\dot{\hat{\theta}}=\operatorname{Proj}(\Gamma \tau)$ in which $\Gamma>0$ is an adaptation gain and $\tau$ is an adaptation function given later, and the projection mapping in (22) guarantees

$$
\begin{aligned}
& \widehat{\theta} \in \Omega_{\widehat{\theta}} \triangleq\left\{\widehat{\theta}: \theta_{\min } \leq \widehat{\theta} \leq \theta_{\max }\right\}, \\
& \widetilde{\theta}^{T}\left(\Gamma^{-1} \operatorname{Proj}_{\widehat{\theta}}(\Gamma \tau)-\tau\right) \leq 0, \quad \forall \tau .
\end{aligned}
$$

Property (P1) implies that the parameter estimation is always within the known bounded set. Property (P2) enables one to show that the use of projection modification to the traditional adaptation law holds the perfect learning capability of the traditional one.

3.3. Normal Robust Controller Design and Results. The design parallels the backstepping procedure [38] with a robust design consideration.

Step 1. Noting that the first equation of (19) does not have any uncertainties, a Lyapunov function can be constructed for the first two equations of (19) directly. Define a switchingfunction-like quantity as

$$
z_{2}=\dot{z}_{1}+k_{1} z_{1}=x_{2}-x_{2 e q}, \quad x_{2 e q} \triangleq \dot{x}_{1 d}-k_{1} z_{1},
$$

where $z_{1}=x_{1}-x_{1 d}(t)$ is the output tracking error and $k_{1}$ is a positive feedback gain. Since $G_{s}(s)=z_{1}(s) / z_{2}(s)=1 /\left(s+k_{1}\right)$ is a stable transfer function, making $z_{1}$ small or converging to zero is equivalent to making $z_{2}$ small or converging to zero. So the rest of the design is to make $z_{2}$ as small as possible with a guaranteed transient performance. Differentiating (24) and noting (19), we have

$$
m \dot{z}_{2}=m \dot{x}_{2}-m \dot{x}_{2 e q}=\bar{x}_{3}-B x_{2}-A_{f} S_{f}\left(x_{2}\right)-\tilde{d}-m \dot{x}_{2 e q} .
$$

In this step, $\bar{x}_{3}$ is treated as virtual control input. Then we can construct a robust control function $\alpha_{2}\left(x_{1}, x_{2}, t\right)$ for the virtual control input $\bar{x}_{3}$ such that output tracking error $z_{1}$ converges to zero or a small value with a guaranteed transient performance. The resulting control function $\alpha_{2}\left(x_{1}, x_{2}, t\right)$ is given by

$$
\begin{gathered}
\alpha_{2}\left(x_{1}, x_{2}, t\right)=\alpha_{2 a}+\alpha_{2 s}, \\
\alpha_{2 a}\left(x_{1}, x_{2}, t\right)=B x_{2}+A_{f} S_{f}\left(x_{2}\right)+m \dot{x}_{2 e q}, \\
\alpha_{2 s}=\alpha_{2 s 1}+\alpha_{2 s 2}, \quad \alpha_{2 s 1}=-k_{2 s 1} z_{2},
\end{gathered}
$$

where $k_{2 s 1}>0$ is a constant gain.
In (26), $\alpha_{2 a}$ functions as a model compensation and $\alpha_{2 s}$ as a robust control law, in which $\alpha_{2 s 2}$ is chosen to satisfy the following conditions:

$$
\begin{aligned}
& \text { Condition (i) } z_{2}\left[\alpha_{2 s 2}-\tilde{d}\right] \leq \varepsilon_{2}, \\
& \text { Condition (ii) } z_{2} \alpha_{2 s 2} \leq 0
\end{aligned}
$$

where $\varepsilon_{2}$ is a positive design parameter which can be arbitrarily small. Essentially, Condition (i) of (27) represents the fact that $\alpha_{2 s 2}$ is synthesized to dominate the uncertain nonlinearities $\widetilde{d}$ with control accuracy measured by the design parameter $\varepsilon_{2}$, and Condition (ii) is to make sure that $\alpha_{2 s 2}$ is dissipating in nature so that it does not interface with the functionality of the model compensation part $\alpha_{2 a}$. How to choose $\alpha_{2 s 2}$ to satisfy constraints like (27) can be found in [27].

Remark 4. One example of a smooth $\alpha_{2 s 2}$ satisfying (27) can be found in the following way. Let $h_{2}$ be any smooth function satisfying

$$
h_{2} \geq \delta_{d}^{2}
$$

then $\alpha_{2 s 2}$ can be chosen as

$$
\alpha_{2 s 2}=-k_{2 s 2} z_{2} \triangleq-\frac{h_{2}}{4 \varepsilon_{2}} z_{2},
$$

where $k_{2 s 2}>0$ is a nonlinear gain. It can be found that (27) is satisfied.

Let $z_{3}=\bar{x}_{3}-\alpha_{2}$ denote the input discrepancy. For the positive-semi-definite (p.s.d.) Lyapunov function $V_{2}$ defined by $V_{2}=m z_{2}^{2} / 2$, noting (25), the time derivative of $V_{2}$ is

$$
\dot{V}_{2}=z_{2}\left[z_{3}+\alpha_{2}-B x_{2}-A_{f} S_{f}\left(x_{2}\right)-\tilde{d}-m \dot{x}_{2 e q}\right]
$$

with the virtual control input (26); then

$$
\dot{V}_{2}=z_{2} z_{3}-k_{2 s 1} z_{2}^{2}+z_{2}\left(\alpha_{2 s 2}-\tilde{d}\right)
$$

Step 2. This step is to synthesize an actual control law for $u$ such that $\bar{x}_{3}$ tracks the virtual control function $\alpha_{2}$ with a guaranteed transient performance as follows. From (19), we can obtain

$$
\dot{z}_{3}=\dot{\bar{x}}_{3}-\dot{\alpha}_{2}=f_{1}(x) u-f_{2}(x)-\eta\left(t-T_{f}\right) \theta f_{3}(x)-\dot{\alpha}_{2},
$$

where

$$
\dot{\alpha}_{2}=\frac{\partial \alpha_{2}}{\partial t}+\frac{\partial \alpha_{2}}{\partial x_{1}} x_{2}+\frac{\partial \alpha_{2}}{\partial x_{2}} \dot{x}_{2}
$$

In (33), the acceleration $\dot{x}_{2}$ is assumed to be available to avoid the propagation of the uncertain nonlinearities $\widetilde{d}$, and thus the conservatism of the robust controller design can be reduced. In the lack of acceleration information case, one can refer to [14], the same general result form (the following Theorem 5) can also be obtained with an enhanced robust controller. 
Based on (32), the final control law can be synthesized by

$$
\begin{gathered}
u=u_{a}+u_{s} \\
u_{a}=\frac{1}{f_{1}(x)}\left[f_{2}(x)+\dot{\alpha}_{2}+\widehat{\theta} f_{3}(x)-z_{2}\right] \\
u_{s}=\frac{1}{f_{1}(x)}\left(u_{s 1}+u_{s 2}\right) \\
u_{s 1}=-k_{3 s 1} z_{3}
\end{gathered}
$$

where $k_{3 s 1}>0$ is a constant gain and $\widehat{\theta}=0$ in (34) for the case where there is no fault or only a slight internal leakage fault.

In (34), $u_{a}$ functions as a model compensation and $u_{s}$ as a robust control law, in which $u_{s 2}$ is chosen as

$$
u_{s 2}=-k_{3 s 2} z_{3} \triangleq-\frac{h_{3}}{4 \varepsilon_{3}} z_{3},
$$

where $\varepsilon_{3}$ is a positive design parameter which can be arbitrarily small and $h_{3}$ is a continuous function satisfying

$$
h_{3} \geq \delta_{q}^{2}(t)
$$

in which $\delta_{q}(t)$ is a positive function for the tolerance level of the slight internal leakage fault; that is, the effect of the slight internal leakage $q_{f}=\eta\left(t-T_{f}\right) \theta f_{3}(x)$ satisfies

$$
\left|q_{f}\right| \leq \delta_{q}
$$

Then the nonlinear robust control law $u_{s 2}$ (35) satisfies the following conditions:

$$
\begin{aligned}
& \text { Condition (i) } z_{3}\left[u_{s 2}-q_{f}\right] \leq \varepsilon_{3}, \\
& \text { Condition (ii) } z_{3} u_{s 2} \leq 0
\end{aligned}
$$

Essentially, Condition (i) of (38) represents the fact that $u_{s 2}$ is synthesized to tolerate the effect of the slight internal leakage $q_{f}$ with a control accuracy measured by the design parameter $\varepsilon_{3}$, and Condition (ii) is to make sure that $u_{s 2}$ is dissipating in nature so that it does not interface with the functionality of the model compensation part $u_{a}$. Define a Lyapunov function as follows:

$$
V_{3}=V_{2}+\frac{1}{2} z_{3}^{2}
$$
is

With the robust control law (34), the time derivative of $V_{3}$

$$
\begin{aligned}
\dot{V}_{3}= & \dot{V}_{2}+z_{3} \dot{z}_{3}=-k_{2 s 1} z_{2}^{2}+z_{2}\left(\alpha_{2 s 2}-\tilde{d}\right) \\
& -k_{3 s 1} z_{3}^{2}+z_{3}\left(u_{s 2}-q_{f}\right) .
\end{aligned}
$$

Thus, we have the following performance theorem.

Theorem 5. In the presence of uncertain nonlinearities satisfying (20b) and/or slight internal leakage fault satisfying (37), the robust control law (34) guarantees the following. (a) In general, all signals are bounded. Furthermore, the p.s.d. Lyapunov function $V_{3}$ is bounded by

$$
V_{3} \leq V_{r}(t)
$$

where

$$
\begin{aligned}
& V_{r}(t)=\exp (-\lambda t) V_{3}(0)+\frac{\varepsilon}{\lambda}[1-\exp (-\lambda t)], \\
& \text { in which } \lambda=2 \min \left\{k_{2 s 1} / m, k_{3 s 1}\right\} \text { and } \varepsilon=\varepsilon_{2}+\varepsilon_{3} .
\end{aligned}
$$

(b) If after a finite time $t_{0}, \tilde{d}=0, q_{f}=0$, that is, in the absence of uncertain nonlinearities and internal leakage fault (i.e., $\widetilde{d}=0, q_{f}=0 \forall t \geq t_{0}$ ), then, in addition to results in (a), asymptotic output tracking is also achieved; that is, $z_{1} \rightarrow 0$ as $t \rightarrow \infty$.

Proof of Theorem 5. From (40), and noting Condition (i) of (27) and (38), then

$$
\begin{aligned}
\dot{V}_{3} & \leq-k_{2 s 1} z_{2}^{2}+\varepsilon_{2}-k_{3 s 1} z_{3}^{2}+\varepsilon_{3} \\
& \leq-\lambda V_{3}+\varepsilon
\end{aligned}
$$

which leads to (41). Thus $z_{1}, z_{2}$ and $z_{3}$ are bounded. Noting Assumption 1 and (20a) and (20b), it follows that $x_{2 e q}$ and the time derivative of $x_{2 e q}$ are bounded; thus $\alpha_{2}$ is bounded, which will lead to the boundedness of $\bar{x}_{3}$. Following Assumption 1, and noting (13), the acceleration $\dot{x}_{2}$ is bounded; thus $\dot{\alpha}_{2}$ is bounded. With Assumption 2, we see that the state $x$ is bounded. The control input $u$ is thus bounded. This proves (a) of Theorem 5 .

Now consider the situation in (b) of Theorem 5, from (40) and noting Condition (ii) of (27) and (38); then

$$
\dot{V}_{3}=-k_{2 s 1} z_{2}^{2}-k_{3 s 1} z_{3}^{2} \leq-\lambda V_{3} .
$$

Since all signals are bounded, following the standard proof procedure in ARC (more details can be found in $[39,40]$ ), it is easy to check that $z_{2} \rightarrow 0$ as $t \rightarrow \infty$, which leads to (b) of Theorem 5.

Remark 6. Results of Theorem 5 indicate that the proposed normal robust controller has an exponentially converging transient performance with the exponentially converging rate $\lambda$ and the final tracking error being able to be adjusted via certain controller parameters freely in a known form; it is seen from (42) that $\lambda$ can be made arbitrarily large, and $\varepsilon / \lambda$, the bound of $V_{3}(\infty)$ (an index for the final tracking errors), can be made arbitrarily small by increasing gains $k_{1}, k_{2 s 1}, k_{3 s 1}$, and/or decreasing controller parameters $\varepsilon_{2}, \varepsilon_{3}$. In this sense, $V_{r}(t)$ can be thought as the prescribed performance index and the actual performance index $V_{3}(t)$ is always governed by the prescribed performance index $V_{r}(t)$ in the normal case and/or the presence of the slight internal leakage fault case. Such a guaranteed transient performance is especially important for the control of electrohydraulic systems since execute time of a run is very short. (b) of Theorem 5 implies that an improved performance can be obtained if there are no fault and modeling errors. 
Remark 7. Designing an extra robust controller $u_{s 2}$ (35) is to emphasize the fact that, if the prescribed control performance $V_{r}(t)$ is maintained even though in the presence of some internal leakage flow, it is not necessary to break off the function of hydraulic systems. And thus abundant unnecessary maintenance cost is saved and the economy is improved for many applications, for instance, machine tools and testing instruments. For aircraft system, this concept can improve the mission availability rate. On the other hand, the effect of the off-line identification errors for some parameters (i.e., $\beta_{e}, C_{l}$, and so on) also can be though as a slight internal leakage fault, and the robust controller can guarantee the control performance with these identification errors.

3.4. Fault Detection and Tolerant Control Design. A performance-oriented fault detection scheme is proposed in this section which is used to detect severe internal leakage fault reliably for preventive maintenance and further fault accommodation (i.e., FTC). The severe internal leakage fault is defined as the violation of the inequality (37) for a period time and simultaneously the control performance is thus deteriorated heavily. The fault detection scheme consists of three components: (1) observed-error generation that indexes the control performance of the controlled hydraulic system; (2) the evaluation scheme that monitors the observed error to detect the presence of any off-nominal system behavior (i.e., decision whether a fault has occurred); and (3) residual generation that gives reliable information for the next fault diagnosis. These three components are detailed as follows.

(1) Observed-Error Generation. The selected observed error must be related to the control performance for the performance-oriented fault detection. It is clear from the normal robust controller design procedure that the p.s.d. Lyapunov function $V_{3}(t)$ is a suitable candidate for this purpose since it is a quadratic form with respect to those control performance indexes $z_{1}, z_{2}$, and $z_{3}$. Thus, we use the performance index $V_{3}(t)$ defined in (39) as the observed error for the fault detection scheme.

(2) The Evaluation Scheme. In order to detect the severe internal leakage fault, we continuously monitor the observederror $V_{3}(t)$ and therefore the threshold for $V_{3}(t)$ has to be so chosen such that, in the absence of the severe fault, $V_{3}(t)$ should be less than the selected threshold for all possible normal cases and/or the slight internal leakage cases; that is, the robustness has to be guaranteed to void any false alarms. From Theorem 5, we recommend the prescribed performance index $V_{r}(t)$ to complete this mission and the robustness of this selected threshold is guaranteed by Theorem 5. The detection decision scheme is given as follows:

$$
I_{m}= \begin{cases}0 & \text { if } V_{3}(t)-V_{r}(t) \leq 0, \\ 1 & \text { if } V_{3}(t)-V_{r}(t)>0,\end{cases}
$$

where $I_{m}$ is the flag whether a fault has occurred or not, $I_{m}=0$ declares that the system is fault-free, and $I_{m}=1$ declares that a fault has been detected.

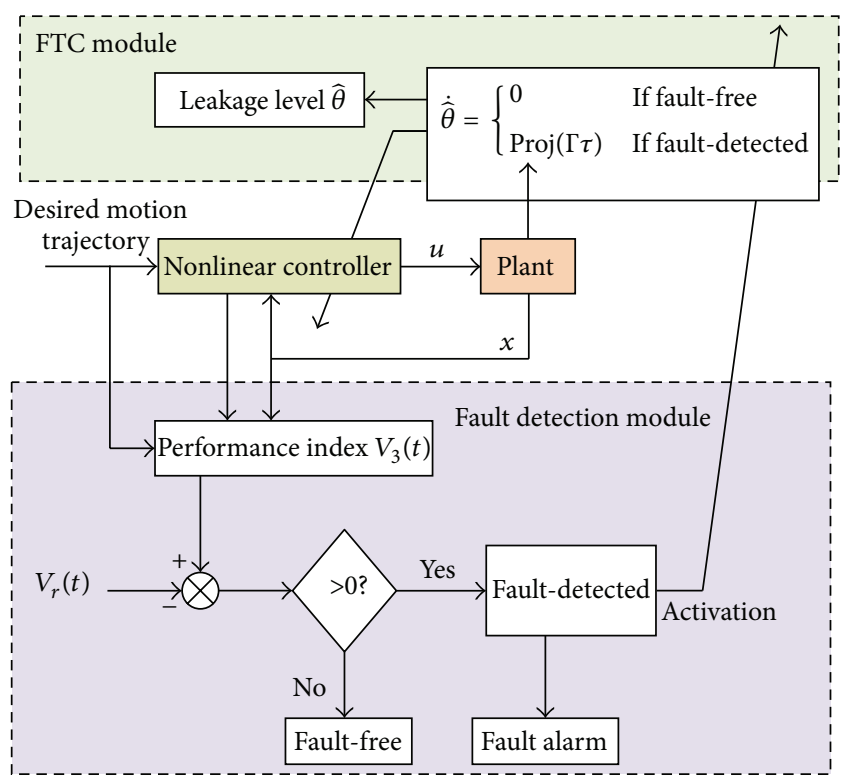

FIgURE 2: The diagram of the proposed fault detection and tolerant control scheme.

(3) Residual Generation. The generation of the residual signal $r(t)$ defined below is used for further fault diagnosis,

$$
r(t)= \begin{cases}0 & \text { fault-free } \\ V_{3}(t) & \text { otherwise }\end{cases}
$$

Since only the internal leakage fault is considered here, the fault diagnosis module is omitted.

At the same time of the detection of a fault, the fault tolerant control designed later is activated immediately.

The overall block diagram of the fault detection scheme, fault tolerant control, and the flow of information in the system are shown in Figure 2. As shown in Figure 2, the proposed fault tolerant control is based on the parameter adaptation scheme. According to the value of $I_{m}$, the learning mechanism is redefined as follows by using the discontinuous projection (22):

$$
\dot{\hat{\theta}}= \begin{cases}0 & \text { if } I_{m}=0 \\ \operatorname{Proj}(\Gamma \tau) & \text { if } I_{m}=1 .\end{cases}
$$

From the properties of the discontinuous projection (22), that is, (23), the learning mechanism is controlled and the parameter estimation is thus retained in the prior known convex set $\Omega_{\theta}$.

The tolerant controller is the same as the aforementioned normal robust controller (34), in which $\hat{\theta}$ is updated by the parameter adaptation law (47) with the initial value $\hat{\theta}(0)=$ 0 . The schematic diagram of the proposed fault tolerant controller is shown in Figure 2.

After the activation of the fault tolerant controller, we have the following theorem.

Theorem 8. With the projection type adaptation law (47) and adaptation function $\tau=\varphi z_{3}$, in which $\varphi=-f_{3}(x)$, 
the proposed fault tolerant controller can gradually recover the control performance; that is, the results of Theorem 5 are restored as $t \rightarrow \infty$, if the following persistent excitation (PE) condition is satisfied:

$$
\int_{t}^{t+T} \varphi^{2}(v) d v \geq \kappa, \quad \text { for some } \kappa>0 \text { and } T>0, \forall t
$$

Proof of Theorem 8. From the fault model (11) and (12), $\eta(t-$ $\left.T_{f}\right) C_{t} \rightarrow C_{t}$ as $t-T_{f} \rightarrow \infty$, thus the third equation of the system model (19) can be written as

$$
\dot{\bar{x}}_{3}=f_{1}(x) u-f_{2}(x)-\theta f_{3}(x)
$$

and the time derivative of $z_{3}$ can be rewritten as

$$
\dot{z}_{3}=\dot{\bar{x}}_{3}-\dot{\alpha}_{2}=f_{1}(x) u-f_{2}(x)-\theta f_{3}(x)-\dot{\alpha}_{2} .
$$

After the activation of the fault tolerant controller, applying the control input $u$ (34) with the adaptation law (47), then

$$
\dot{z}_{3}=-z_{2}-k_{3 s 1} z_{3}+u_{s 2}-\tilde{\theta} \varphi
$$

It is clear from (51) that there is only parametric uncertainty $\widetilde{\theta}$ and no unmodeled disturbances. Therefore, it is well known that the parameter estimation converges to its true value (i.e., $\widetilde{\theta} \rightarrow 0$ as $t \rightarrow \infty$ ) if the PE condition (48) is satisfied [41]. Then the effect of the fault is counteracted by the added term in the fault tolerant controller (i.e., the adaptive compensation term $\left.\hat{\theta} f_{3}(x)\right)$. That is to say, as $t \rightarrow \infty$, the time derivative of $V_{3}(t)$ satisfies

$$
\begin{aligned}
\dot{V}_{3} & \leq-k_{2 s 1} z_{2}^{2}+\varepsilon_{2}-k_{3 s 1} z_{3}^{2}+z_{3} u_{s 2} \\
& \leq-\lambda V_{3}+\varepsilon
\end{aligned}
$$

which will lead to the result (a) of Theorem 5. If there are no uncertain nonlinearities in the entire system, (i.e., $\widetilde{d}=0$ ), from (52) and the proof procedure of Theorem 5, we have

$$
\dot{V}_{3} \leq-\lambda V_{3}
$$

which will lead to the result (b) of Theorem 5 . Thus the results of Theorem 5 are gradually recovered.

Remark 9. It is clear from (48) that the PE condition is easily satisfied as the integrated term is a square of a scalar function $\varphi$. Thus the demand of the richness of the motion trajectory is greatly relaxed and the parameter adaptation is prone to implement. The proposed tolerant controller is also prone to realize as only a slight modification has to be made based on the normal robust controller (34). Furthermore, the fault leakage level can be caught by the excellent convergence of the parameter adaptation scheme.

Remark 10. Based on the proposed fault detection scheme (45) and the fault tolerant controller (34) with (47), one can assign the required control performance based on the results of Theorem 5, and the tolerance level of the internal leakage (i.e., $\delta_{q}$ ) via certain controller parameters freely in a known form. Small value of $\delta_{q}$ means the high sensitivity of the internal leakage fault. Large value of $\delta_{q}$, on the contrary, means the high robustness of the internal leakage fault.

\section{Simulation Results}

To illustrate the above designs, simulation results are obtained for the hydraulic system discussed in Section 2, that is, (1) (8), and (10). Since the double-rod hydraulic actuators are widely discussed [28, 31, 32], for more conveniently and easily comprehending the basis idea of the proposed fault detection and tolerant control scheme, in this section, the double-rod hydraulic actuator, which is a particular case of the single-rod hydraulic actuator, is used for the illustrative purpose. Another reason of choosing the doublerod hydraulic actuator as the plant to be discussed is that we only have a dual-vane hydraulic rotary actuator which is equivalent to the double-rod hydraulic actuator in the schematic sense in our laboratory. Moreover, it is clear from Section 3 that the type of the hydraulic actuator does not influence the verification of the effectiveness of the proposed fault detection and control scheme. The nominal equivalent parameters of the hydraulic actuator are summarized in Table 1 which have been identified in detail.

The following two controllers are compared.

(1) NRC: the nonlinear robust controller (34) without fault accommodation, that is, $\widehat{\theta} \equiv 0$ no matter the severe leakage fault occurs or not. The controller parameters are listed in Table 2.

(2) FTC: the proposed fault tolerant controller (34) with adaptation law (47), in which $\theta_{\min }=0, \theta_{\max }=$ $6 \times 10^{-7}, \Gamma=1 \times 10^{-21}$. From the parameters listed in Table 2, it can be seen that the converging rate $\lambda=100$. Choosing $\delta_{d}=7200$ means that about $1 \mathrm{~L} / \mathrm{min}$ internal leakage fault flow can be tolerant in the following tests.

In the following simulation tests, $x_{1 d}=$ $\arctan (0.2 \sin (2 \pi t))\left[1-\exp \left(-0.1 t^{3}\right)\right] \mathrm{m}$ is used as the motion trajectory which satisfies Assumption 1, and thus $V_{3}(0)=0$.

To test the effectiveness of the proposed fault detection and tolerant control algorithms, three typical cases of the internal leakage fault are considered: Case 1 characterizes the abrupt internal leakage fault; Case 2 characterizes the incipient internal leakage fault; Case 3 characterizes the slight internal leakage fault. The parameters of the internal leakage fault are listed in Table 3.

For Case 1, the fault detection is shown in Figure 3 (the index $V_{3}$ is chopped off). It is clear that the fault is immediately detected ( $I_{m}=1$ when $\left.t=6 \mathrm{~s}\right)$ when it occurs $\left(T_{f}=6 \mathrm{~s}\right.$ ) as the inequality (41) is immediately violated by the abrupt severe internal leakage fault. After the detection of the abrupt fault, the FTC controller is activated and the control performance is gradually restored which is shown in Figure 4. If one compares FTC with NRC, it is seen that, before the detection of the abrupt fault, the FTC and NRC are the same. Along with the activation of the parameter adaptation, the FTC can gradually counteract the effect of the fault and the serious performance deterioration of NRC is saved by FTC. The excellent convergence of the parameter estimation is given in Figure 5 which can indicate the level of the fault. The results of the control input are present in Figure 6, which 
TABLE 1: Nominal equivalent parameters of the considered hydraulic system.

\begin{tabular}{llc}
\hline Symbol & \multicolumn{1}{c}{ Parameter } & Nominal value \\
\hline$m$ & The total mass of the load & $40 \mathrm{~kg}$ \\
$A_{1}$ & The ram area of the cylinder & $1.92 \times 10^{-4} \mathrm{~m}^{2}$ \\
$A_{2}$ & The ram area of the cylinder & $1.92 \times 10^{-4} \mathrm{~m}^{2}$ \\
$B$ & The coefficient of the viscous friction & $45 \mathrm{~N} \cdot \mathrm{s} / \mathrm{m}$ \\
$f_{c}$ & The Coulomb friction & $52 \mathrm{~N}$ \\
$f_{s}$ & The stiction friction & $78 \mathrm{~N}$ \\
$\dot{y}_{v}$ & The Stribeck velocity & $0.013 \mathrm{~m} / \mathrm{s}$ \\
$\sigma$ & The empirical parameter of the Stribeck effect & 2 \\
$\beta_{e}=\beta_{1}=\beta_{2}$ & The bulk modulus of the oil in the cylinder & $200 \mathrm{Mpa}$ \\
$V_{01}$ & The original total control volume of the cylinder & $1.76 \times 10^{-4} \mathrm{~m}^{3}$ \\
$V_{02}$ & The original total control volume of the cylinder & $1.76 \times 10^{-4} \mathrm{~m}{ }^{3}$ \\
$C_{l}$ & The normal coefficient of the internal leakage & $8.9 \times 10^{-12} \mathrm{~m}^{5} / \mathrm{Ns}$ \\
$k_{q}=k_{q 1}=k_{q 2}$ & The flow gain of the servovalve & $9.92 \times 10^{-6} \mathrm{~m}^{4} /(\mathrm{s} \cdot \mathrm{A} \cdot \sqrt{\mathrm{N})}$ \\
$P_{s}$ & The supply pressure & $7 \mathrm{Mpa}$ \\
$P_{r}$ & The return pressure & $0 \mathrm{Mpa}$ \\
$\omega_{v}$ & The servovalve natural frequency & $628 \mathrm{rad} / \mathrm{s}$ \\
$\zeta_{v}$ & The servovalve damping ratio & 0.7 \\
$k_{i}$ & The gain of the servovalve & $4 \times 10^{-3} \mathrm{~A} / \mathrm{V}$ \\
\hline
\end{tabular}

TABLE 2: The parameters of the proposed control scheme.

\begin{tabular}{|c|c|c|}
\hline Symbol & Parameter & Value \\
\hline$A_{f}$ & The amplitude of the Coulomb friction & $52 \mathrm{~N}$ \\
\hline$S_{f}$ & The continuous shape function & $\arctan (900 \dot{y})$ \\
\hline$k_{1}$ & The feedback gain for $z_{1}$ & 10 \\
\hline$k_{2 s 1}$ & The feedback gain for $z_{2}$ & 2000 \\
\hline$\delta_{d}$ & $\begin{array}{l}\text { The bound of the uncertain } \\
\text { nonlinearities } \widetilde{d}\end{array}$ & $100 \mathrm{~N}$ \\
\hline$\varepsilon_{2}$ & The controller parameter for $\alpha_{2 s 2}$ & 1 \\
\hline$k_{3 s 1}$ & The feedback gain for $z_{3}$ & 50 \\
\hline$\delta_{q}$ & $\begin{array}{l}\text { The tolerance level of the internal } \\
\text { leakage fault }\end{array}$ & 7200 \\
\hline$\varepsilon_{3}$ & The controller parameter for $u_{s 2}$ & $1 \times 10^{5}$ \\
\hline
\end{tabular}

TABLE 3: The parameters of the internal leakage fault.

\begin{tabular}{lccc}
\hline & Case 1 & Case 2 & Case 3 \\
\hline$C_{t}\left(\mathrm{~m}^{4} /(\mathrm{s} \cdot \sqrt{\mathrm{N}})\right)$ & $4.5 \times 10^{-7}$ & $1 \times 10^{-7}$ & $1.2 \times 10^{-8}$ \\
$\mu$ & 10 & 0.5 & 5 \\
$T_{f}(\mathrm{~s})$ & 6 & 6 & 6 \\
\hline
\end{tabular}

show that the control input of FTC and NRC is close to the maximum authority of the control input $(10 \mathrm{~V})$ due to the severe internal leakage fault. In this case, the maximum flow of the internal leakage is $38 \mathrm{~L} / \mathrm{min}$, which is $60 \%$ of the load flow of the servovalve $(63 \mathrm{~L} / \mathrm{min})$.

For Case 2, that is, the incipient internal leakage fault which develops very slowly, the fault detection is shown in Figure 7. The fault is detected $\left(I_{m}=1\right.$ when $\left.t=6.33 \mathrm{~s}\right)$ after a period of its occurrence $\left(T_{f}=6 \mathrm{~s}\right)$ as the fault is slowly developing and the effect of the fault between $t=6 \mathrm{~s}$ and
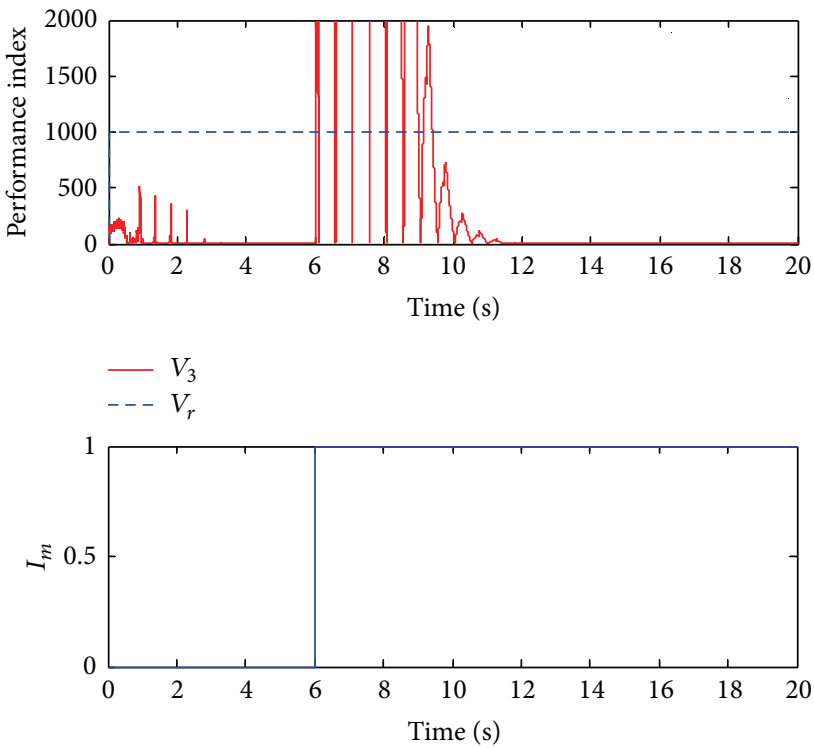

FIGURE 3: Case 1: fault detection.

$t=6.33 \mathrm{~s}$ is tolerant by the prior given bound $\delta_{q}$. After the detection of the incipient fault, the FTC controller is activated and the control performance is gradually restored which is shown in Figure 8. The parameter estimation is given in Figure 9 and the level of the incipient fault is indicated. The results of the control input are present in Figure 10. Although the control effort of NRC and FTC is almost the same, the FTC controller makes a more accurate accommodation than that of the NRC controller. From the above results, it can be seen that the proposed scheme can effectively detect the fault which will destroy the control performance, that is, inequality (41). In the early developing period, the fault is 

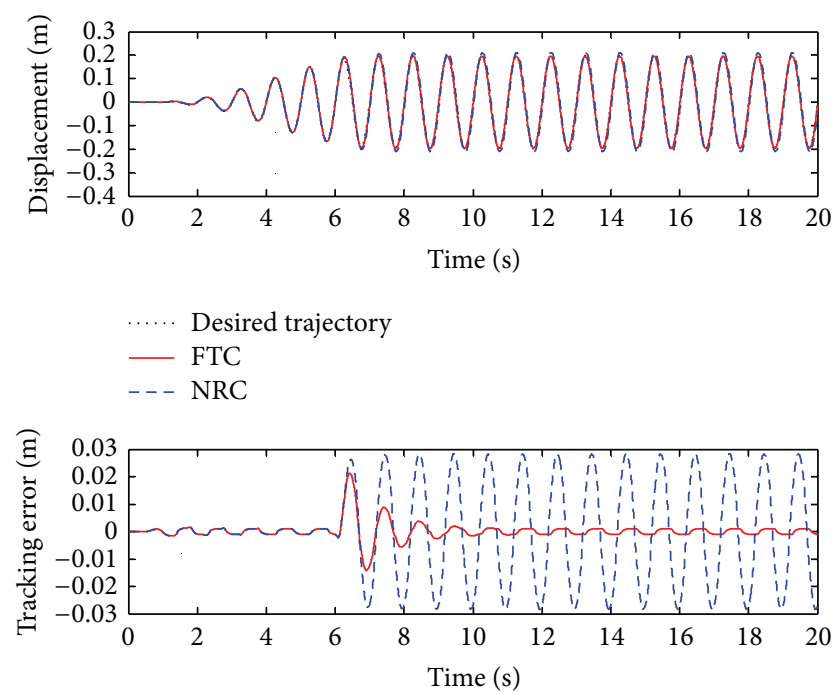

- FTC

--- NRC

FIGURE 4: Case 1: tracking errors of FTC and NRC.

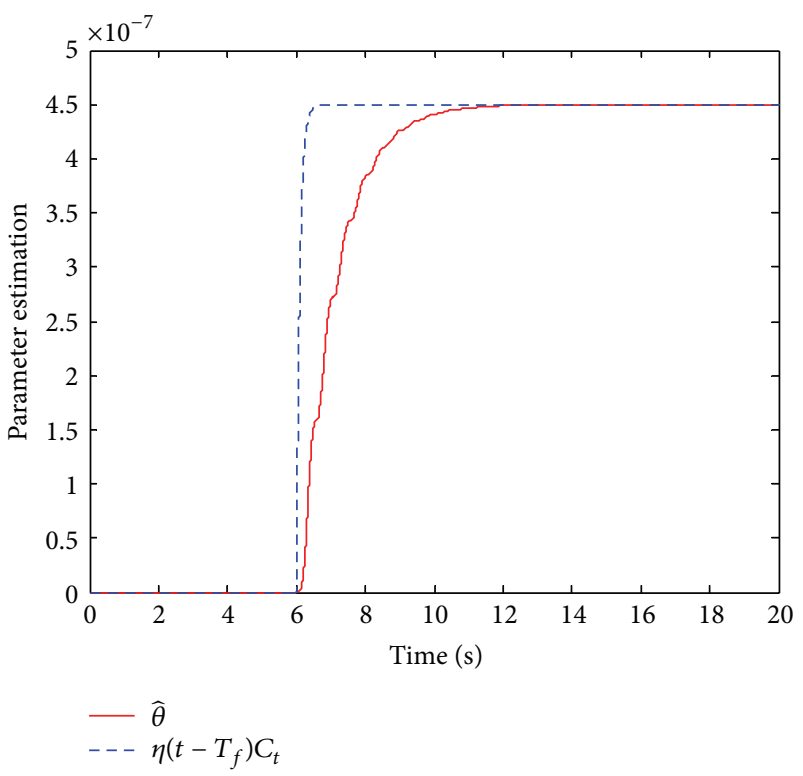

FIgURE 5: Case 1: parameter estimation.

small enough such that it does not influence the control performance; hence, it is not necessary to detect it. But once it develops largely enough and begins to deteriorate the tracking performance, the proposed scheme detected immediately and activated the FTC to compensate the fault and recover the control performance.

For Case 3, the effect of the slight internal leakage fault does not exceed the threshold $\delta_{q}$; that is, the inequality of (37) is respected which is shown in Figure 11. The control performance of NRC does not obviously deteriorate which is shown in Figure 12. In this case, the fault is very small such that the control performance is not affected. Hence,

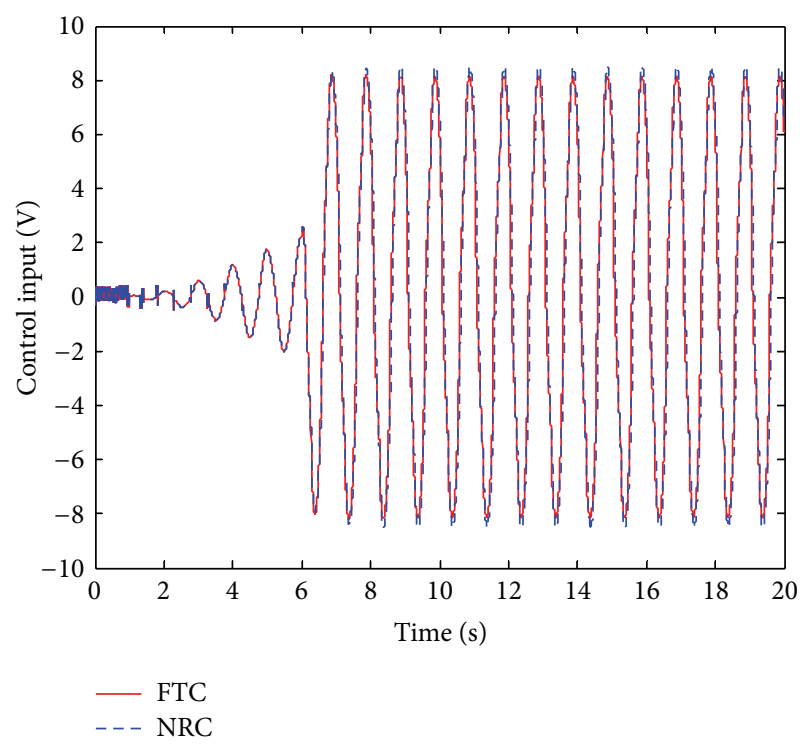

FIgURE 6: Case 1: the control input of FTC and NRC.
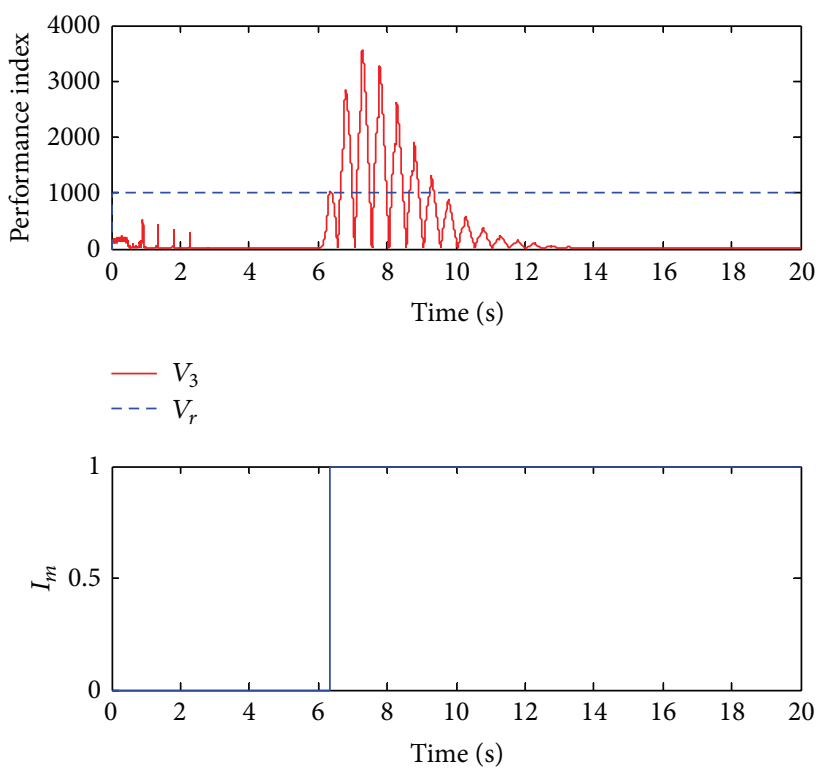

Figure 7: Case 2: fault detection.

the fault is not necessary to be alarmed. So no fault is detected $\left(I_{m}=0\right.$ for all $\left.t\right)$ though the slight fault has occurred $\left(T_{f}=6 \mathrm{~s}\right)$, which is given in Figure 13. In this case, the FTC controller is the same as the NRC controller. The control input is present in Figure 14. The results of Case 3 verify the prior design of the tolerance of the slight fault, that is, the bound $\delta_{d}$. The unnecessary maintenance and the breakoff of hydraulic systems can be avoided for this slight fault. Following this design concept, the maintenance cost is reduced and the economy is improved; simultaneously the control performance is also guaranteed, that is, just the purpose of the proposed FDD scheme. The idea of the tolerance of the slight fault might make a better trade-off 

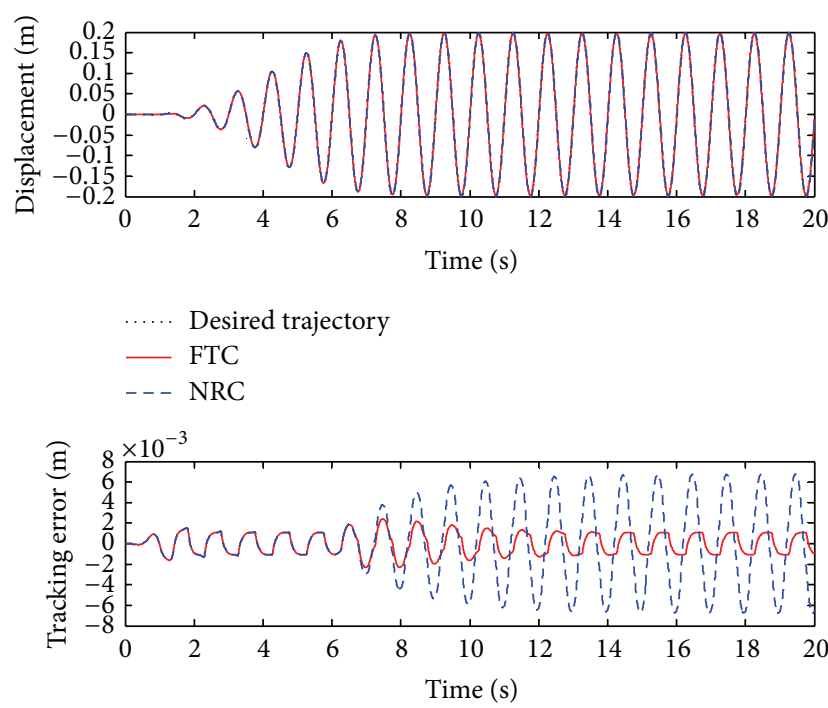

- FTC

- - NRC

FIGURE 8: Case 2: tracking errors of FTC and NRC.

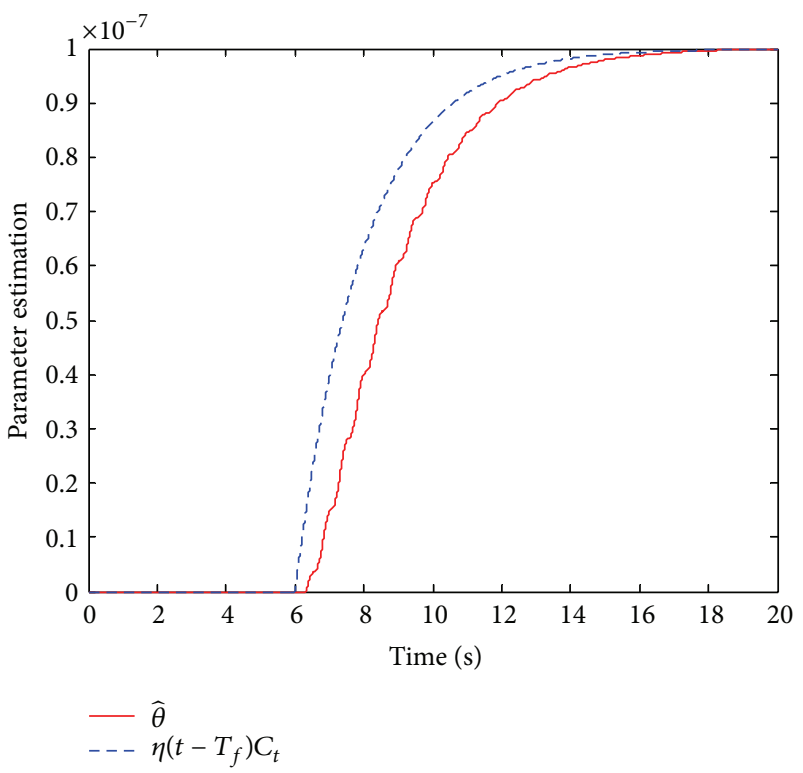

FIGURE 9: Case 2: parameter estimation.

between the performance and the economy of the hydraulic control systems.

\section{Conclusions}

In this paper, a novel fault detection method is proposed which is a performance-oriented scheme. The main feature of the scheme is that a tolerance level of the slight fault is given, and the control performance of the hydraulic systems is guaranteed by a normal robust controller in the normal case and/or the presence of the slight fault case. For the severe fault, the detection scheme gives a reliable

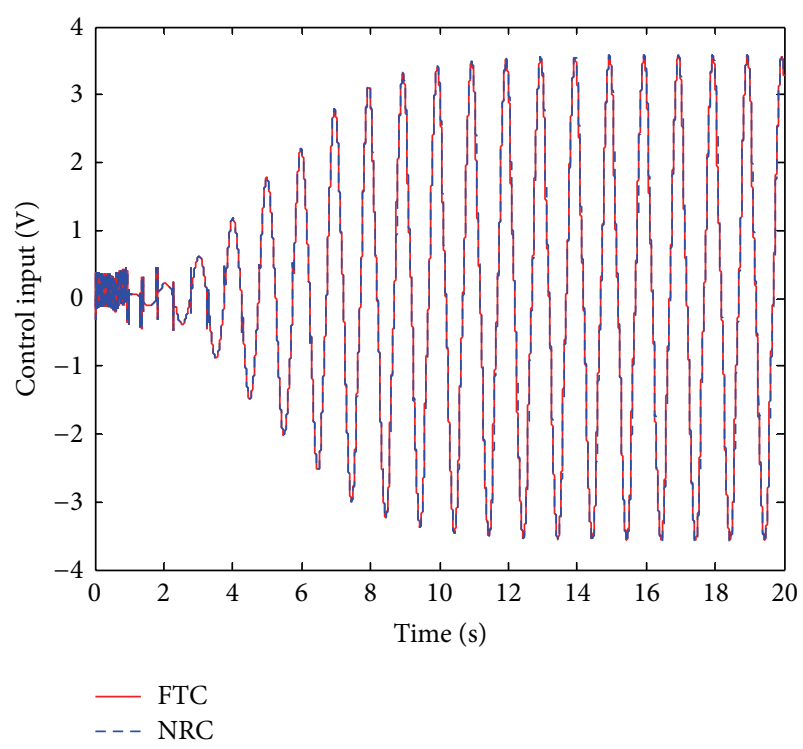

FIGURE 10: Case 2: control input of FTC and NRC.

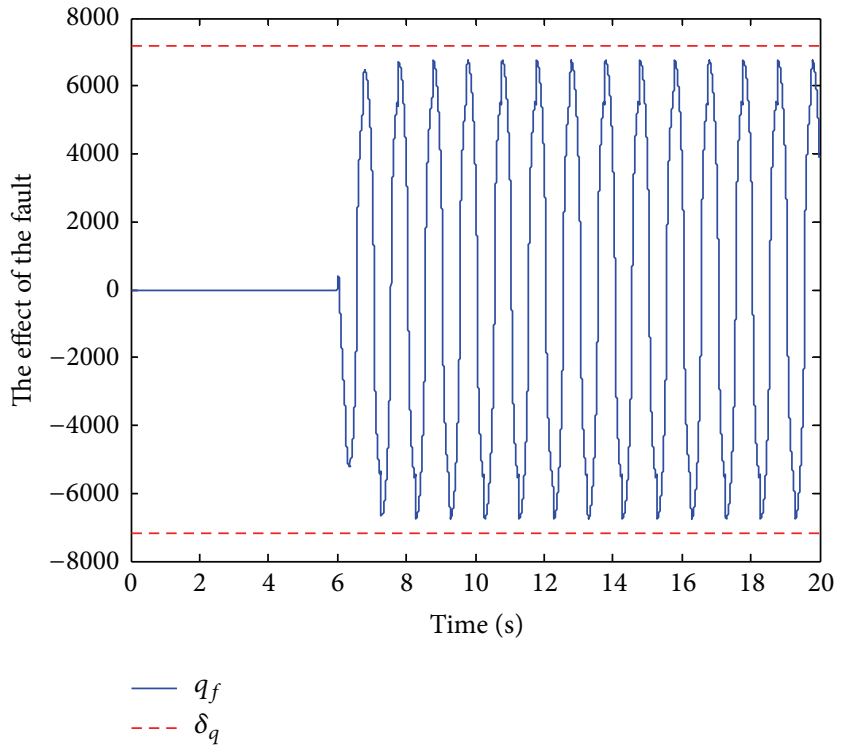

Figure 11: Case 3: the effect of the slight internal leakage fault.

detection. The concept of the proposed fault detection may reduce the maintenance cost and the economy can be improved with the hydraulic control systems. Based on this fault detection scheme, the internal leakage fault is accommodated by the proposed fault tolerant controller via the parameter estimation. Since only the coefficient of the internal leakage fault is considered and other parameters are identified off-line and the identification errors can be lumped into the unmodeled nonlinearities, the PE condition is easily satisfied which gives an excellent convergence of the parameter estimation. Then the level of the fault can be provided. After the activation of the fault tolerant controller, the control performance is gradually restored. In addition, the fault tolerant controller has a simple structure which is 

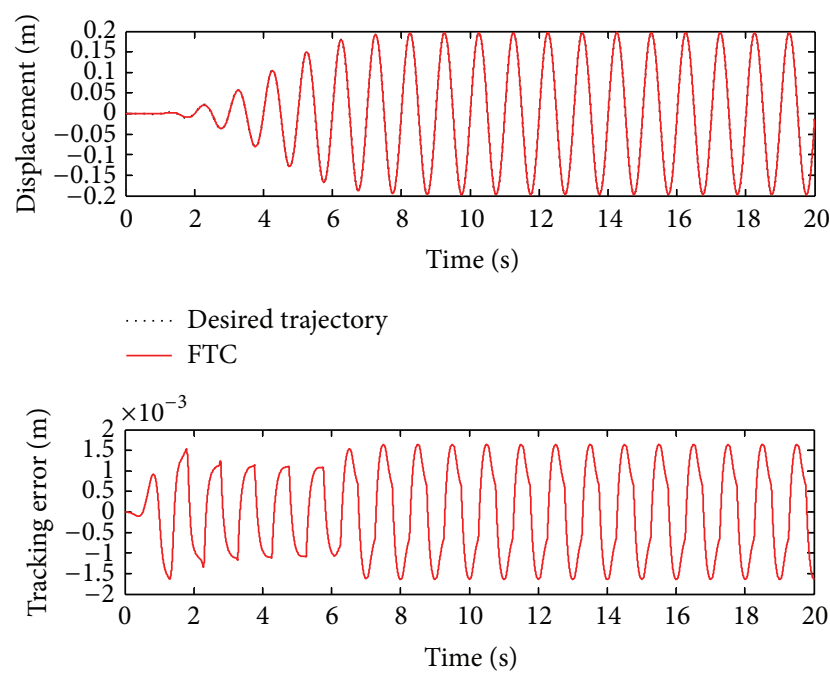

FIGURE 12: Case 3: tracking errors of NRC.
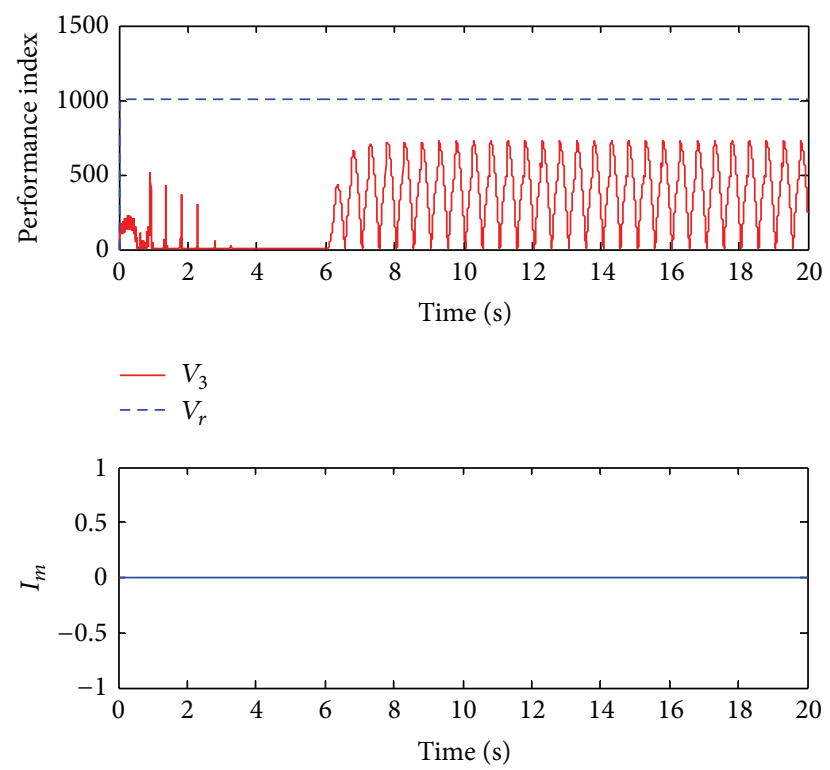

FIgURE 13: Case 3: fault detection.

based on the normal robust controller; thus the computing time is saved. Another contribution of the proposed fault detection and tolerant control scheme is that one can assign the required control performance and the tolerance level of the internal leakage via certain controller parameters freely in a known form. Three cases of the internal leakage fault, that is, the abrupt severe fault, the incipient fault, and the slight fault, are investigated to verify the effectiveness of the proposed fault detection and tolerant control strategy. From the model development, it can be seen that the synthesized model is particularly conservative, which might lead the proposed scheme to be inappropriate for physical hydraulic systems. In the future, a more general and comprehensive fault detection and tolerant control should be considered for hydraulic servo systems, with various parametric uncertainties and uncertain

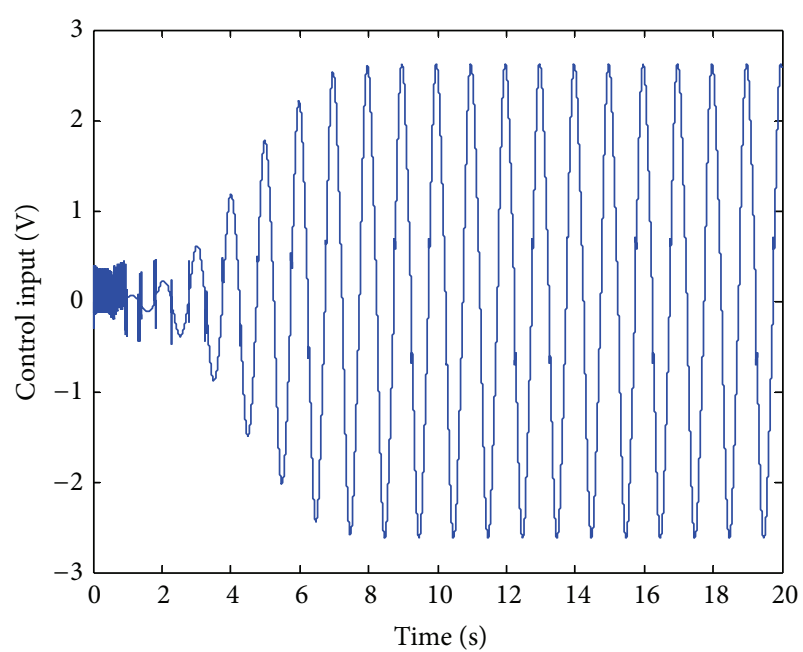

FIGURE 14: Case 3: the control input of NRC.

nonlinearities, to remove the prerequisites utilized in this paper.

\section{Conflict of Interests}

The authors declare that there is no conflict of interests regarding the publication of this paper.

\section{Acknowledgments}

The first author is grateful to Professor B. Yao for hosting his visit at Purdue University from October 2010 to October 2011 and the guidance in ARC design. This work was supported in part by the National Natural Science Foundation of China (Grant no. 51305203), in part by the Specialized Research Fund for the Doctoral Program of Higher Education (Grant no. 20133219120026), China, and in part by Jiangsu Planned Projects for Postdoctoral Research Funds (Grant no. 1302002A).

\section{References}

[1] W. H. Zhu and J. C. Piedboeuf, "Adaptive output force tracking control of hydraulic cylinders with applications to robot manipulators," ASME Journal of Dynamic Systems, Measurement and Control, vol. 127, no. 2, pp. 206-217, 2005.

[2] B. Yao, M. Al-Majed, and M. Tomizuka, "High-performance robust motion control of machine tools: an adaptive robust control approach and comparative experiments," IEEE/ASME Transactions on Mechatronics, vol. 2, no. 2, pp. 63-76, 1997.

[3] W. Sun, H. Gao, and B. Yao, "Adaptive robust vibration control of full-car active suspensions with electrohydraulic actuators," IEEE Transactions on Control Systems Technology, vol. 21, no. 6, pp. 2417-2422, 2013.

[4] W. Sun, H. Gao, and O. Kaynak, "Adaptive backstepping control for active suspension systems with hard constraints," IEEE/ ASME Transactions on Mechatronics, vol. 18, no. 3, pp. 10721079, 2013. 
[5] J. Yao, Z. Jiao, D. Ma, and L. Yan, "High-accuracy tracking control of hydraulic rotary actuators with modeling uncertainties," IEEE/ASME Transactions on Mechatronics, vol. 19, no. 2, pp. 633-641, 2014.

[6] C. Guan and S. Pan, "Nonlinear adaptive robust control of single-rod electro-hydraulic actuator with unknown nonlinear parameters," IEEE Transactions on Control Systems Technology, vol. 16, no. 3, pp. 434-445, 2008.

[7] J. Yao, Z. Jiao, and S. Han, "Friction compensation for low velocity control of hydraulic flight motion simulator: a simple adaptive robust approach," Chinese Journal of Aeronautics, vol. 26, no. 3, pp. 814-822, 2013.

[8] J. Yao, Z. Jiao, and D. Ma, "Extended-state-observer-based output feedback nonlinear robust control of hydraulic systems with backstepping," IEEE Transactions on Industrial Electronics, 2014.

[9] J. Yao, Z. Jiao, and B. Yao, "Robust control for static loading of electro-hydraulic load simulator with friction compensation," Chinese Journal of Aeronautics, vol. 25, no. 6, pp. 954-962, 2012.

[10] M. Karpenko and N. Sepehri, "Hardware-in-the-loop simulator for research on fault tolerant control of electrohydraulic actuators in a flight control application," Mechatronics, vol. 19, no. 7, pp. 1067-1077, 2009.

[11] J. Yao, Z. Jiao, B. Yao, Y. Shang, and W. Dong, "Nonlinear adaptive robust force control of hydraulic load simulator," Chinese Journal of Aeronautics, vol. 25, no. 5, pp. 766-775, 2012.

[12] N. Sepehri, M. Karpenko, L. An, and S. Karam, "A test rig for experimentation on fault tolerant control and condition monitoring algorithms in fluid power systems: from design through implementation," Transactions of the Canadian Society for Mechanical Engineering, vol. 29, no. 3, pp. 441-458, 2005.

[13] H. E. Merritt, Hydraulic Control Systems, Wiley, New York, NY, USA, 1967.

[14] B. Yao, F. Bu, J. Reedy, and G. T.-C. Chiu, "Adaptive robust motion control of single-rod hydraulic actuators: theory and experiments," IEEE/ASME Transactions on Mechatronics, vol. 5, no. 1, pp. 79-91, 2000.

[15] R. Isermann, "On the design and control of mechatronic systems-a survey," IEEE Transactions on Industrial Electronics, vol. 43, no. 1, pp. 4-15, 1996.

[16] J. Watton, Condition Monitoring and Fault Diagnosis in Fluid Power Systems, Ellis Horwood, Chichester, UK, 1992.

[17] D. Yu, "Fault diagnosis for a hydraulic drive system using a parameter-estimation method," Control Engineering Practice, vol. 5, no. 9, pp. 1283-1291, 1997.

[18] H. Z. Tan and N. Sepehri, "Parametric fault diagnosis for electrohydraulic cylinder drive units," IEEE Transactions on Industrial Electronics, vol. 49, no. 1, pp. 96-106, 2002.

[19] P. M. Frank, "Online fault detection in uncertain nonlinear systems using diagnostic observers: a survey," International Journal of System Science, vol. 25, no. 12, pp. 2129-2154, 1994.

[20] H. Hammouri, P. Kabore, S. Othman, and J. Biston, "Failure diagnosis and nonlinear observer. Application to a hydraulic process," Journal of the Franklin Institute, vol. 339, no. 4-5, pp. 455-478, 2002.

[21] M. Munchhof, Model-based fault detection for a hydraulic servo axis [Ph.D. thesis], Technischen Univestitat Darmstadt, Darmstadt, Germany, 2006.

[22] L. An and N. Sepehri, "Hydraulic actuator circuit fault detection using extended Kalman filter," in Proceedings of the American Control Conference, pp. 4261-4266, Denver, Colo, USA, June 2003.
[23] R. Rajamani and J. K. Hedrick, "Adaptive observers for active automotive suspensions: theory and experiment," IEEE Transactions on Control Systems Technology, vol. 3, no. 1, pp. 86-93, 1995.

[24] P. Garimella and B. Yao, "Model based fault detection of an electro-hydraulic cylinder," in Proceedings of the American Control Conference (ACC'05), pp. 484-489, Portland, Ore, USA, June 2005.

[25] P. Garimella and B. Yao, "Nonlinear adaptive robust observer design for a class of nonlinear systems," in Proceedings of the American Control Conference, pp. 4391-4396, Denver, Colo, USA, June 2003.

[26] S. Gayaka and B. Yao, "Fault detection, identification and accommodation for an electro-hydraulic system: an adaptive robus," in Proceedings of the 17th World Congress, International Federation of Automatic Control (IFAC'08), pp. 13815-13820, Seoul, Republic of Korea, July 2008.

[27] B. Yao and M. Tomizuka, "Adaptive robust control of siso nonlinear systems in a semi-strict feedback form," Automatica, vol. 33, no. 5, pp. 893-900, 1997.

[28] M. Karpenko and N. Sepehri, "Robust position control of an electrohydraulic actuator with a faulty actuator piston seal," ASME Journal of Dynamic Systems, Measurement and Control, vol. 125, no. 3, pp. 413-423, 2003.

[29] L. An and N. Sepehri, "Hydraulic actuator leakage fault detection using extended Kalman filter," International Journal of Fluid Power, vol. 6, no. 1, pp. 41-51, 2005.

[30] A. Y. Goharrizi and N. Sepehri, "A wavelet-based approach to internal seal damage diagnosis in hydraulic actuators," IEEE Transactions on Industrial Electronics, vol. 57, no. 5, pp. 17551763, 2010.

[31] M. Karpenko and N. Sepehri, "Fault-tolerant control of a servohydraulic positioning system with crossport leakage," IEEE Transactions on Control Systems Technology, vol. 13, no. 1, pp. 155-161, 2005.

[32] M. Karpenko and N. Sepehri, "Quantitative fault tolerant control design for a leaking hydraulic actuator," ASME Journal of Dynamic Systems, Measurement and Control, vol. 132, no. 5, pp. 237-244, 2010.

[33] C. S. Tzafestas, G. G. Rigatos, and S. G. Tzafestas, "Design of fault-tolerant control systems: passive and active approaches," Systems Science, vol. 24, no. 4, pp. 5-28, 1998.

[34] B. Armstrong-Hélouvry, P. Dupont, and C. C. De Wit, "A survey of models, analysis tools and compensation methods for the control of machines with friction," Automatica, vol. 30, no. 7 , pp. 1083-1138, 1994.

[35] A. Alleyne and R. Liu, "A simplified approach to force control for electro-hydraulic systems," Control Engineering Practice, vol. 8, no. 12, pp. 1347-1356, 2000.

[36] D. F. Thompson, J. S. Pruyn, and A. Shukla, "Feedback design for robust tracking and robust stiffness in flight control actuators using a modified QFT technique," International Journal of Control, vol. 72, no. 16, pp. 1480-1497, 1999.

[37] X. Zhang, M. M. Polycarpou, and T. Parisini, "A robust detection and isolation scheme for abrupt and incipient faults in nonlinear systems," IEEE Transactions on Automatic Control, vol. 47, no. 4, pp. 576-593, 2002.

[38] M. Krstic, I. Kanellakopoulos, and P. V. Kokotovic, Nonlinear and Adaptive Control Design, Wiley, New York, NY, USA, 1995.

[39] J. Yao, G. Yang, Z. Jiao, and D. Ma, "Adaptive robust motion control of direct-drive dc motors with continuous friction 
compensation," Abstract and Applied Analysis, vol. 2013, Article ID 837548, 14 pages, 2013.

[40] J. Yao, Z. Jiao, and D. Ma, "Adaptive robust control of DC motors with extended state observer," IEEE Transactions on Industrial Electronics, vol. 61, no. 7, pp. 3630-3637, 2014.

[41] B. Yao and C. Jiang, "Advanced motion control: from classical PID to nonlinear adaptive robust control," in Proceedings of the 11th IEEE International Workshop on Advanced Motion Control (AMC'10), pp. 815-829, Nagaoka, Japan, March 2010. 


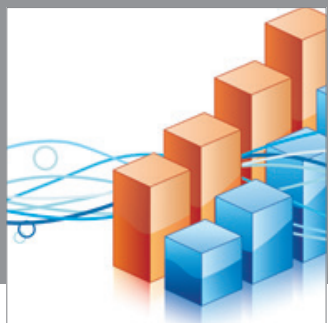

Advances in

Operations Research

mansans

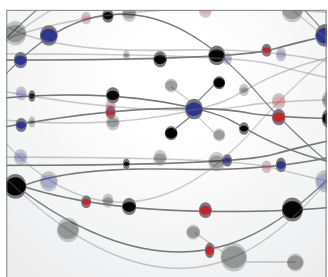

The Scientific World Journal
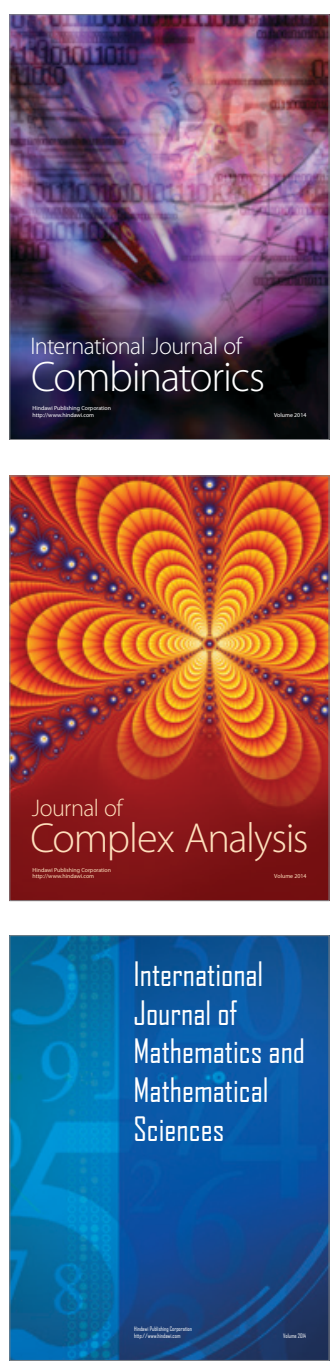
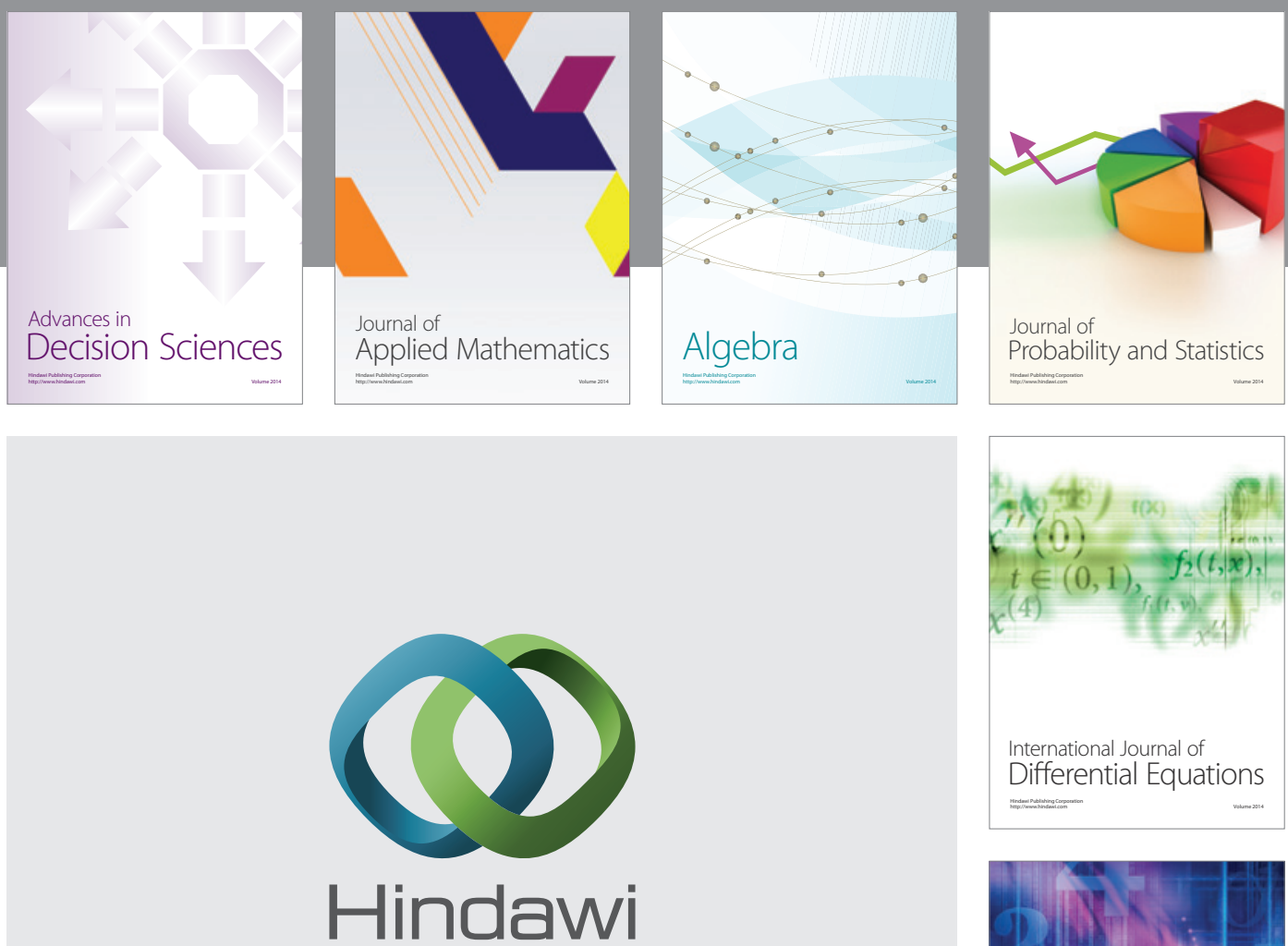

Submit your manuscripts at http://www.hindawi.com
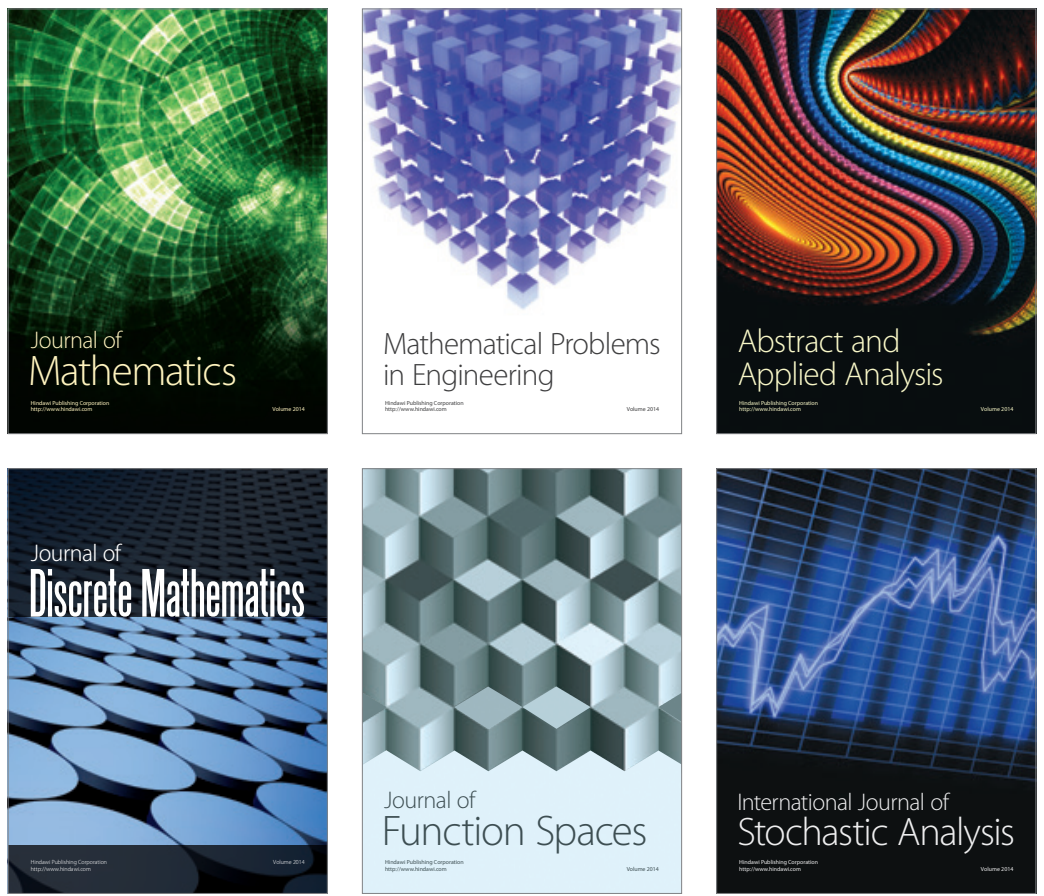

Journal of

Function Spaces

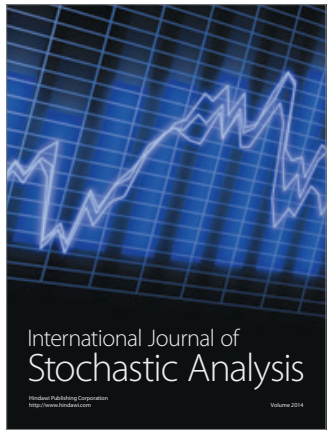

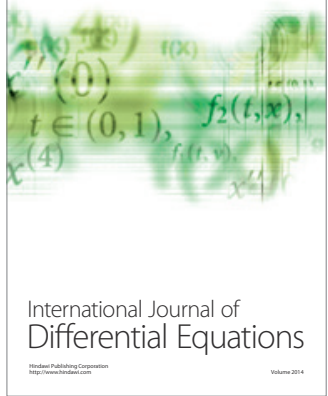
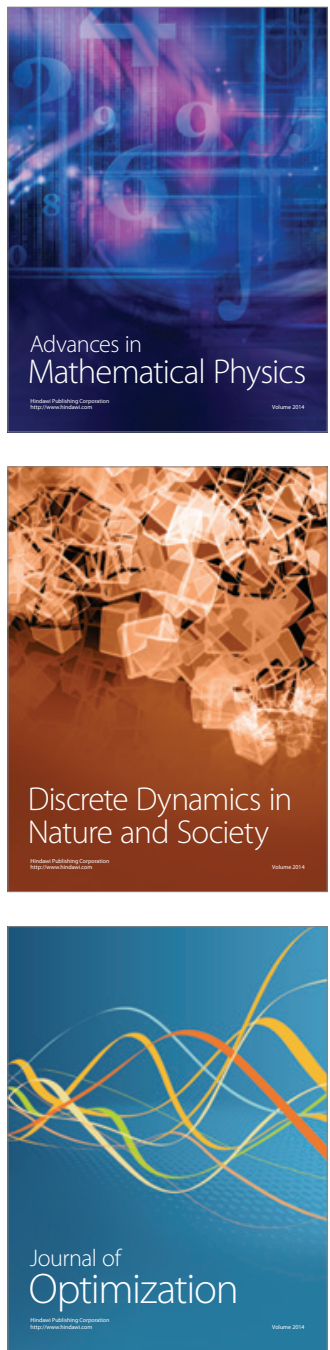